\title{
Three-dimensional modelling of the human carotid artery using the lattice Boltzmann method. II: Shear analysis
}

\author{
J Boyd $\nmid$ and J M Buick $\ddagger$ \\ $\dagger$ Cardiovascular Research Group, Physics, University of New England, Armidale, \\ NSW, 2351, Australia \\ $\ddagger$ Mechanical \& Design Engineering, Anglesea Building, Anglesea Road, University of \\ Portsmouth, Portsmouth, PO1 3DJ UK
}

\begin{abstract}
.
Near wall shear is known to be important in the pathogenesis and progression of atherosclerosis. In this paper the shear field in a three-dimensional model of the human carotid artery is presented. The simulations are performed using the lattice Boltzmann model and are presented at six times of interest during a physiologically accurate velocity waveform. The near wall shear rate and von Mises effective shear are also examined. Regions of low near wall shear rates are observed near the outer wall of the bifurcation and in the lower regions of the external carotid artery. These are regions where low near wall velocity and circulatory flows have been observed and are regions that are typically prone to atherosclerosis.
\end{abstract}

PACS numbers: 47.11.-j, 47.11.Qr, 47.63.-b, 87.19.U-

Submitted to: Phys. Med. Biol.

\section{Introduction}

Atherosclerotic cardiovascular disease is a leading cause of morbidity in the industrialised world $[1,2]$. There is a body of evidence that suggests a correlation between atherosclerosis, regions of low blood flow velocity, circulating flow and low shear stress near the artery walls $[3,4,5]$. Therefore the study of the haemodynamic properties of the blood in these regions of the artery can lead to a greater understanding of atherosclerosis and its dependence on flow parameters.

Here the lattice Boltzmann model (LBM) $[6,7,8]$ is used to simulate the human carotid artery and the shear inside the artery and near the artery walls are considered. Details of the numerical method and the carotid artery simulations are presented in detail in a companion paper [9]. Here only a brief description of the key components will be given. Details of the velocity in the artery model are also presented in [9]. 


\section{Theoretical Background}

The simulations were performed using the lattice Boltzmann method [6]. This is a simplified kinetic model which evolves on a regular underlying grid. Here the threedimensional D3Q15 lattice is used. This is a cubic grid where each grid point is connected to 14 neighbours along link directions $\mathbf{e}_{i}$ where $i$ labels the link and

$$
\begin{array}{ll}
\mathbf{e}_{0} & =(0,0,0), \\
\mathbf{e}_{1,2}, \mathbf{e}_{3,4}, \mathbf{e}_{5,6} & = \pm(1,0,0), \pm(0,1,0), \pm(0,0,1), \text { and }, \\
\mathbf{e}_{7,13}, \mathbf{e}_{8,14}, \mathbf{e}_{9,11}, \mathbf{e}_{10,12} & = \pm(1,1,1), \pm(-1,1,1), \pm(-1,-1,1), \pm(1,-1,1) .
\end{array}
$$

Both the fluid velocity and shear can be determined directly from the model at each grid point to second-order accuracy. In particular it is not necessary to differentiate the velocity field to obtain the shear.

The artery geometry is presented in [9] and also in figure 1. The region of interest around the bifurcation is indicated in part (a) of the figure while part (b) shows the plane slices through the artery on which the results will be presented. These correspond to the velocity results presented in [9]. The regions are labelled $1-11$ from the bottom slice and are spaced approximately $3.4 \mathrm{~mm}$ (24 lattice points) apart in the $z$ direction. The common carotid artery (CCA), internal carotid artery (ICA) and external carotid artery (ECA) are indicated by the arrows in both figures.

(a)

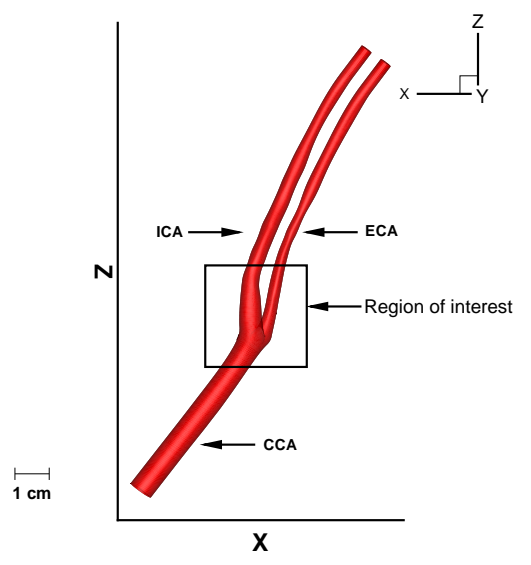

(b)

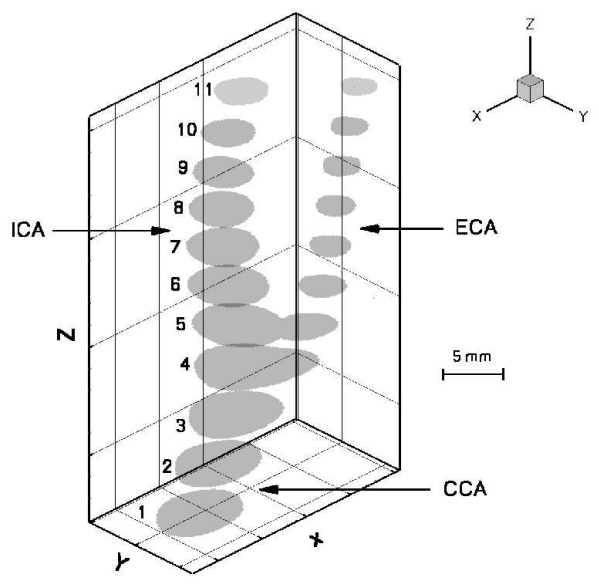

Figure 1. (a) Carotid artery geometry with region of interest around the bifurcation indicated, (b) Plane slices through the bifurcation, labelled 1 - 11. Slices are spaced approximately $3.4 \mathrm{~mm}$ apart in the $Y$ direction. In both images the common carotid artery (CCA), internal carotid artery (ICA) and external carotid artery (ECA) are indicated.

Simulations were performed in parallel [10] on the 16 processor University of New England Beowulf cluster. A pulsatile waveform adapted from Holdsworth et al (1999) [11] is shown in figure 2. A sub-grid accurate extrapolation boundary scheme [12] is used which retains the second order nature of the LBM and enables the shape of the 


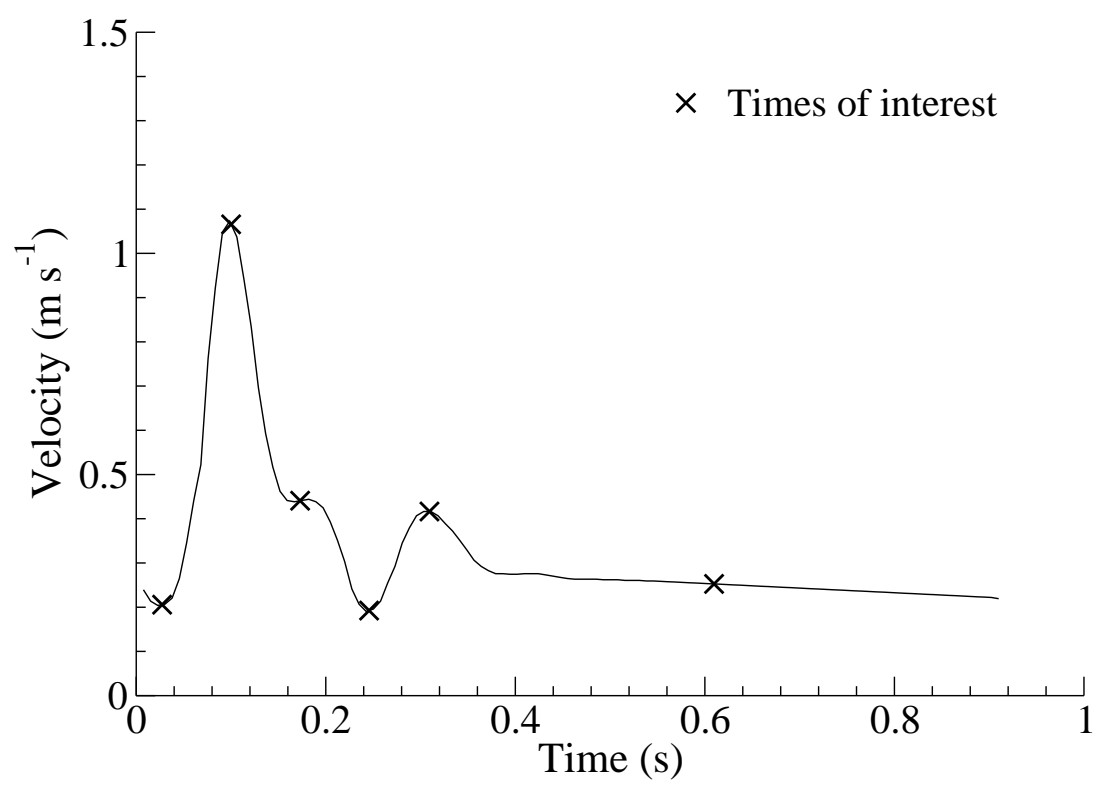

Figure 2. Pulse waveform adapted from [11], with times of interest indicated. Times from left to right are $t=0.03 \mathrm{~s}, t=0.1 \mathrm{~s}, t=0.17 \mathrm{~s}, t=0.24 \mathrm{~s}, t=0.31 \mathrm{~s}$ and $t=0.6 \mathrm{~s}$.

artery wall to be modelled at a resolution greater than that provided by the underlying lattice $[13,14,15]$. This is illustrated in figure 3 where the link $\mathbf{e}_{\mathbf{w}}$ between the fluid node $\mathbf{x}_{f}$ and the external node $\mathbf{x}_{e}$ is intersected by the curved artery boundary at $\mathbf{x}_{b}$. To determine the state of the fluid at the node $\mathbf{x}_{f}$ the LBM uses the fluid properties at adjacent nodes, including $\mathbf{x}_{e}$ at the previous time-step. Since $\mathbf{x}_{e}$ is not in the fluid the extrapolation boundary scheme [12] determines fictitious fluid properties at the external node. These are found using $\Delta$, the distance between $\mathbf{x}_{f}$ and $\mathbf{x}_{b}$ as well as the known fluid properties at $\mathbf{x}_{f}$ and $\mathbf{x}_{f f}$.

\section{Results}

In this paper two invariants of the shear tensor will be examined. The first is the shear rate $\dot{\gamma}$, defined as

$$
\dot{\gamma}=2 \sqrt{D_{I I}},
$$

where

$$
D_{I I}=\sum_{\alpha, \beta=1}^{3} S_{\alpha \beta} S_{\alpha \beta} .
$$

The second was the von Mises effective stress [16, 17], defined as

$$
\sigma_{e f f}=\sqrt{\frac{A+6 B}{2}},
$$


where

$$
\begin{aligned}
& A=\left(\sigma_{x x}-\sigma_{y y}\right)^{2}+\left(\sigma_{y y}-\sigma_{z z}\right)^{2}+\left(\sigma_{x x}-\sigma_{z z}\right)^{2}, \text { and } \\
& B=\sigma_{x y}^{2}+\sigma_{y z}^{2}+\sigma_{x z}^{2} .
\end{aligned}
$$

Both of these invariants are orientation independent, and can capture much information about the local shear environment in the artery. In addition they can be easily calculated at each lattice node, without having to rely on the local velocity field gradients (see $[9,16]$ ).

The shear stress was determined at each lattice grid point and was then used to calculate $\dot{\gamma}$ and $\sigma_{\text {eff }}$, equations (2) and (4) respectively. This information was then used to calculate $\dot{\gamma}$ and $\sigma_{\text {eff }}$ on the wall of the artery and one grid length from the wall using a linear extrapolation from the nearest and next nearest lattice nodes in the same link direction.

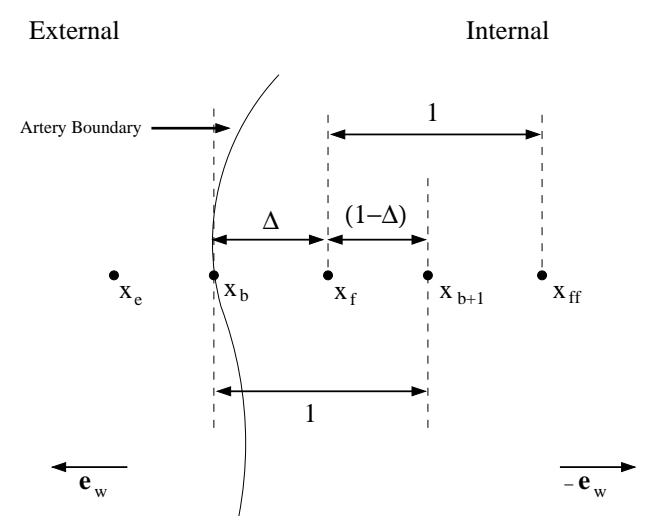

Figure 3. Extrapolation points used for the sub-grid boundary scheme and near wall shear analysis. $x_{b}$ represents a point on the boundary of the artery, which is a distance $\Delta$ from the closest fluid node $x_{f} . x_{f f}$ is the next fluid node after $x_{f}$ in the same link direction, and $x_{b+1}$ is a point in the fluid one lattice unit away from the artery wall. $x_{e}$ represents a grid node external to the artery.

Figure 3 illustrates this method. For each fluid node $x_{f}$ adjacent to the artery boundary, that is the nodes for which the adjacent node $x_{e}$ in some link direction $\mathbf{e}_{w}$ is external to the artery; the next closest fluid node in the link direction $-\mathbf{e}_{w}, x_{f f}$, was found. The distance of the artery boundary from $x_{f}$ in lattice units is given by $\Delta \in(0,1)$, the same parameter which is applied in the extrapolation boundary scheme. Since both $\dot{\gamma}$ and $\sigma_{\text {eff }}$ are known at $x_{f}$ and $x_{f f}$, these values can be used to linearly extrapolate to find the shear rate on the boundary, $\dot{\gamma}\left(x_{b}\right)$ and interpolate to find the shear rate one grid length in, $\dot{\gamma}\left(x_{b+1}\right)$, along the link direction. Extrapolations and interpolations were only taken along the axial lattice link directions, i.e. $\mathbf{e}_{1}, \ldots, \mathbf{e}_{6}$. 
The extrapolations and interpolations were performed in the following manner,

$$
\begin{aligned}
& \dot{\gamma}\left(x_{b}\right)=\dot{\gamma}\left(x_{f}\right)-\left[\dot{\gamma}\left(x_{f f}\right)-\dot{\gamma}\left(x_{f}\right)\right] \Delta, \text { and } \\
& \dot{\gamma}\left(x_{b+1}\right)=\dot{\gamma}\left(x_{f}\right)+\left[\dot{\gamma}\left(x_{f f}\right)-\dot{\gamma}\left(x_{f}\right)\right](1-\Delta) .
\end{aligned}
$$

The values of $\sigma_{e f f}\left(x_{b}\right)$ and $\sigma_{e f f}\left(x_{b+1}\right)$ were found using the same method. The known values $\dot{\gamma}\left(x_{f}\right)$ and $\sigma_{e f f}\left(x_{f}\right)$, that is, the calculated values on the fluid nodes nearest to the artery boundary without extrapolation, were also considered in order to compare the extrapolated and interpolated values.

(a)

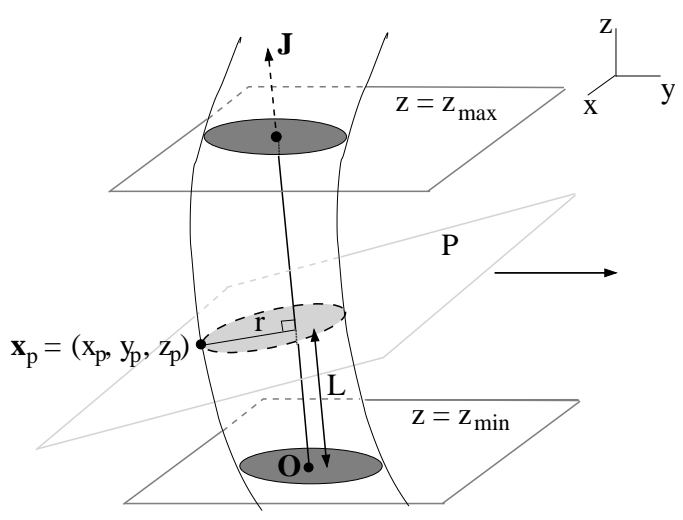

(b)

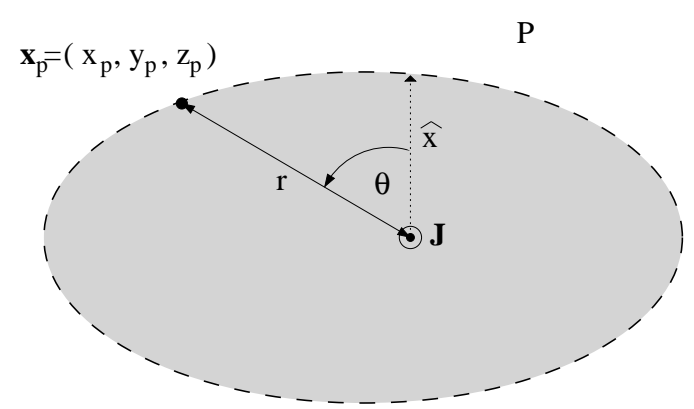

Figure 4. Defining regions and parameters used in the projection method.

The wall shears were mapped into a two-dimensional plane using the projection method shown in figures 4 (a) and (b). The regions of analysis were confined to relatively straight sections of the artery around the bifurcation. The curvature of the artery section shown in figure 4 (a) has been exaggerated to illustrate this technique. Figure 5 shows the three regions of the carotid artery bifurcation that this analysis was performed on. Region 1 corresponds to the ICA, region 2 corresponds to the ECA and region 3 corresponds to the CCA.

These regions were chosen to lie between planes defined by $z_{\min }$ and $z_{\max }$. The cross-sectional areas of the artery segment defined by the interactions of these planes with the artery, indicated in dark gray in figure 4 (a), were found and their centers were then joined by a straight line whose vector direction is labelled $\mathbf{J}$. The center on the plane $z=z_{\text {min }}$ was taken as the local origin $O$. All points of interest $\mathbf{x}_{p}=\left(x_{p}, y_{p}, z_{p}\right)$ within a region $z_{\text {min }} \leq z_{p} \leq z_{\text {max }}$ were then determined. Here the points of interest corresponded to either the boundary points $\mathbf{x}_{b}$, the fluid points closest to the artery boundary $\mathbf{x}_{f}$ or the points one lattice grid length from the boundary $\mathbf{x}_{b+1}$; see figure 3. Points $\mathbf{x}_{f}$ are lattice grid points, but points $\mathbf{x}_{b}$ and $\mathbf{x}_{b+1}$ are not on the lattice. The plane $\mathrm{P}$ passing through $\mathbf{x}_{p}$ with normal $\mathbf{J}$ was then found. The portion of the plane inside the artery is light gray in figure 4 (a) and shown in more detail in figure 4 (b). 


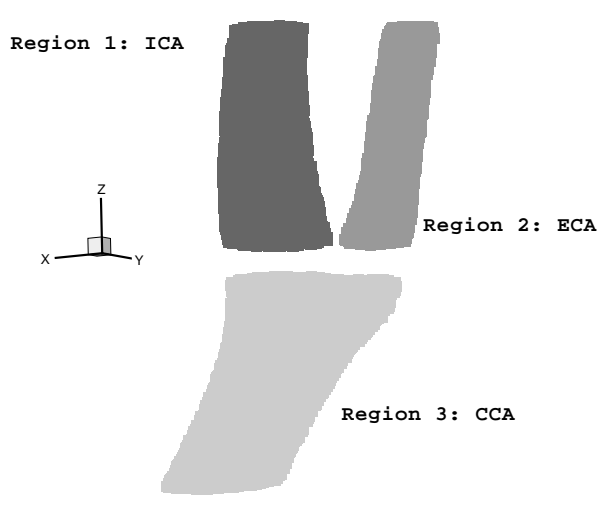

Figure 5. Regions of the carotid bifurcation where wall shear stress was calculated. Region 1 corresponds to the ICA, Region 2 corresponds to the ECA and Region 3 corresponds to the CCA.

The position of $\mathbf{x}_{p}$ was then expressed as a function of $L, r$ and $\theta$, where $L$ is the path length along $J$ between the planes $z=z_{\min }$ and $P ; r$ is the magnitude of the vector

$$
\mathbf{r}=\mathbf{x}_{p}-\frac{L \mathbf{J}}{\|\mathbf{J}\|},
$$

and $\theta$ is the angle between $\mathbf{r}$ and $\hat{\mathbf{x}}$,

$$
\theta=\frac{\cos ^{-1}(\mathbf{r} \cdot \hat{\mathbf{x}})}{\|\mathbf{r}\|},
$$

where $\hat{\mathbf{x}}$ is a unit vector in the direction of the projection of the $x$-axis into the plane $P$. This is shown in figure 4 (b). $\dot{\gamma}$ and $\sigma_{\text {eff }}$ were then expressed in terms of the co-ordinate system $(r, L, \theta)$ for each of the three extrapolation schemes.

Figures 6 (a) - (c) show the normalised radius distributions in regions 1 - 3 of the carotid artery bifurcation respectively. In these figures the radius distributions are normalised by the peak radius in each region. Peak radii corresponded to $0.0037 \mathrm{~m}$ in region $1,0.00225 \mathrm{~m}$ in region 2 , and $0.005 \mathrm{~m}$ in region 3 . Here the radius distribution indicates two features of the artery, namely the irregularity of the artery and the extent to which it is curved. For example, consider the artery geometry depicted in figure 4 (a). From figure 4 (b) we see that $r$ has a maximum at $\theta \simeq 90^{\circ}$ and a minimum at $\theta \simeq 270^{\circ}$. This behaviour is evident in figure 6 (a) indicating a degree of curvature in the artery, see figures 1 and 5. This is also evident to a lesser extent in region 2, see figure 6 (b). Figure 6 (c) shows a different feature. Here, towards the bottom of the region, the radius has a maximum close to $\theta=90^{\circ}$ and $\theta=270^{\circ}$ and a minimum around $\theta=180^{\circ}$ and $\theta=360^{\circ}$ indicating that the artery cross-section is elliptical here, prior to the bifurcation. This continues along the length of the region before becoming obscured close to the bifurcation. In regions 1 and 2 a general reduction in the radius is observed with increasing length, while in region 3 the radius increases towards the 
(a)

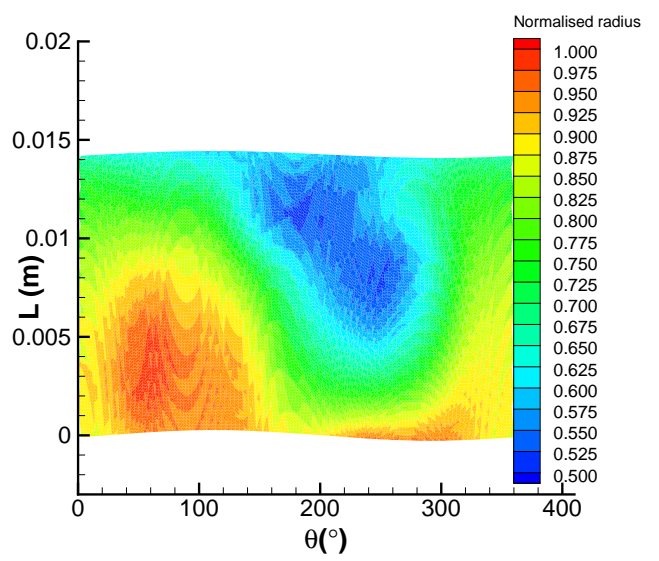

(b)

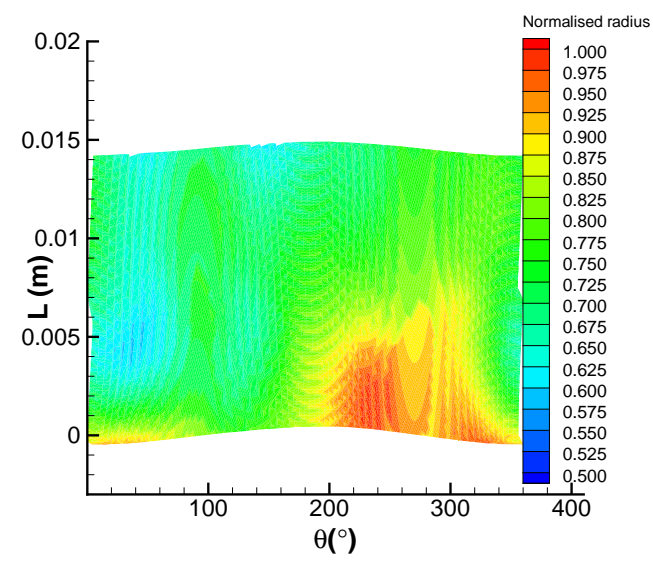

(c)

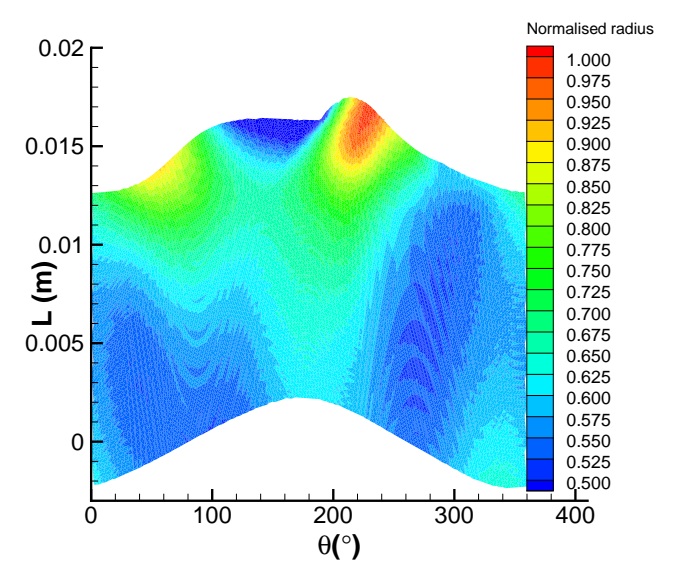

Figure 6. Normalised radius distributions in (a) region 1, (b) region 2, and (c) region 3 of the carotid bifurcation.

bifurcation. This general trend along the length of each region is more significant than any irregularities in the artery wall.

The calculated $(r, L, \theta)$ values for $\dot{\gamma}$ and $\sigma_{e f f}$ form a non-regular data set. In order to represent the data it was triangulated using Tecplot 10 [18], a commercial plotting program.

Results for the on wall extrapolation (points $\mathbf{x}_{b}$ ), no extrapolation (points $\mathbf{x}_{f}$ ) and one lattice length from the wall interpolation (points $\mathbf{x}_{b+1}$ ) showed only small differences throughout the pulse cycle. All wall results exhibit a slight lack of smoothness, see for example figure 7. This was due to the data triangulation and is not present in the direct plots of the shear, see for example figure 8 (d), or the velocity results [9]. Despite this, sufficient resolution is achieved such that solid statements about the wall shear behaviour can be made. Due to the negligible differences observed between the extrapolation and interpolation schemes, only the analysed data without extrapolation will be presented here. Use of this data will reduce any errors associated with using a linear extrapolation or interpolation schemes. 
(a)

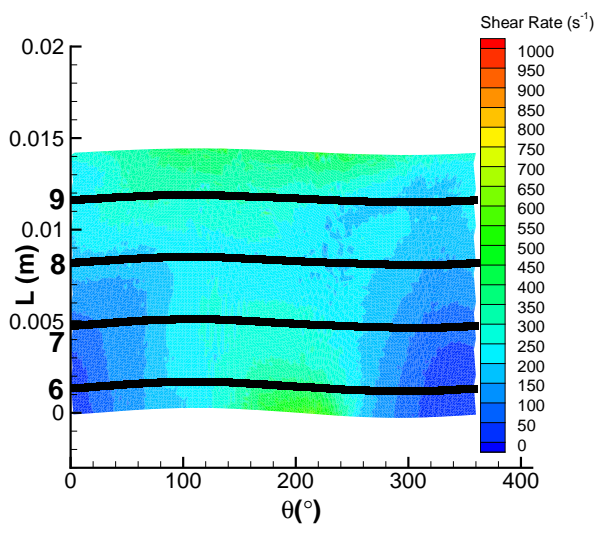

(b)
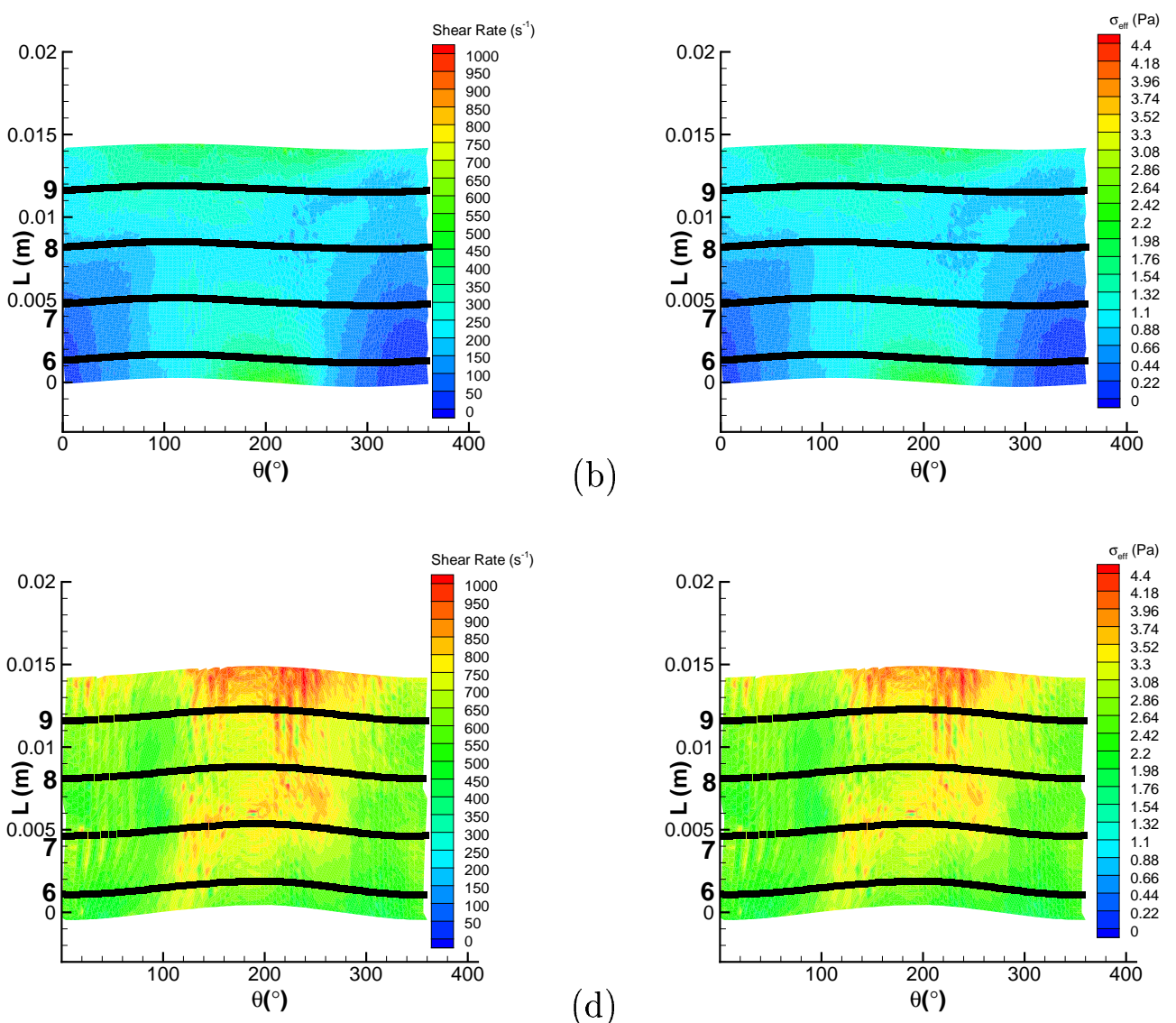

(c)

(e)

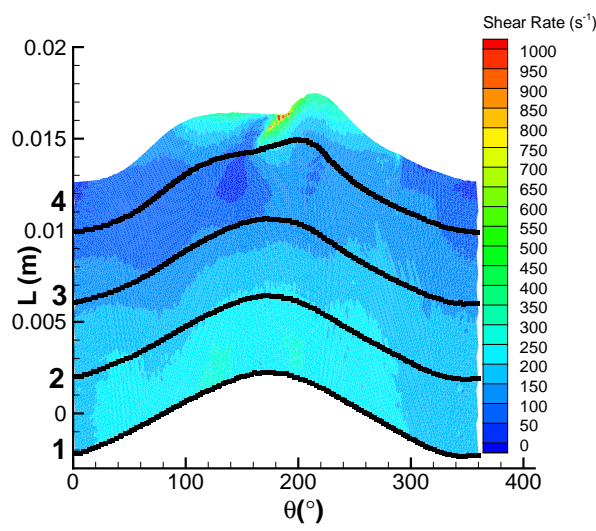

(d)

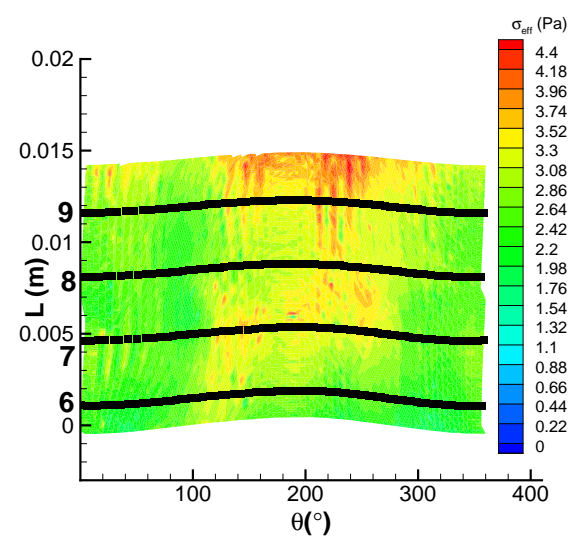

(f)

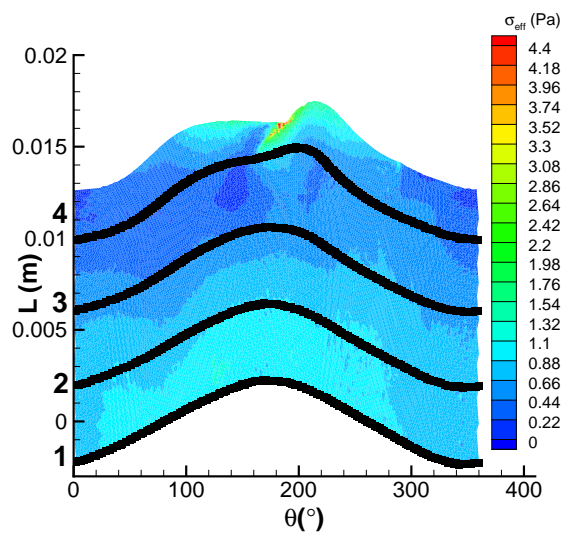

Figure 7. Comparison of shear rate, $\dot{\gamma}$, and the von Mises effective stress $\sigma_{e f f}$ at time $t=0.31 \mathrm{~s}$. (a) - (b) region 1, (c) - (d) region 2 and (e) - (f) region 3 shown. The numbered black lines correspond to the plane slices labelled in figure 1 (b). 
Figures 7 (a) - (f) show comparisons of $\dot{\gamma}$ and $\sigma_{\text {eff }}$ at time $t=0.31 \mathrm{~s}$ in regions 1 ( (a) - (b) ), 2 ( (c) - (d) ) and 3 ( (e) - (f) ). The black lines in these figures, numbered $1-4$ in figures 7 (e) - (f) and $6-9$ in figures 7 (a) - (d) correspond to the numbered plane slices seen in figure 1 (b). It is observed that the wall $\dot{\gamma}$ and $\sigma_{\text {eff }}$ compare very well qualitatively, with the main difference being the scale of the results. Both $\dot{\gamma}$ and $\sigma_{\text {eff }}$ show the same general features over the whole of the pulse cycle in all regions. Therefore only results for $\dot{\gamma}$ will be presented in this paper. It is noted that the magnitude of the shears compare well to literature results $[13,19,20,21]$.

(a)

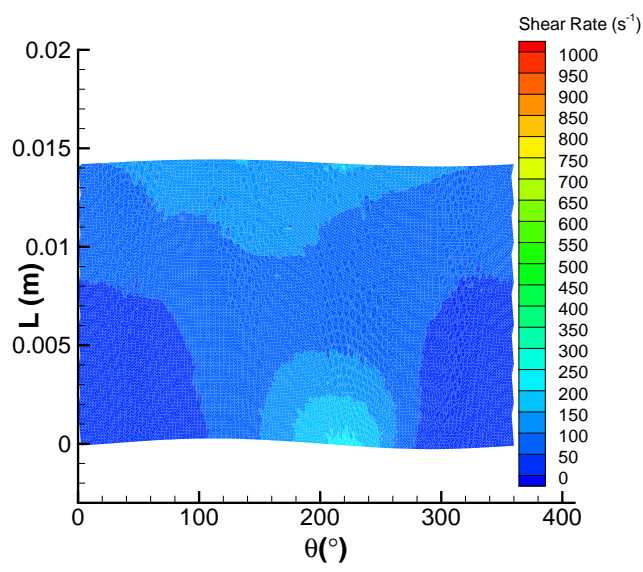

(c)

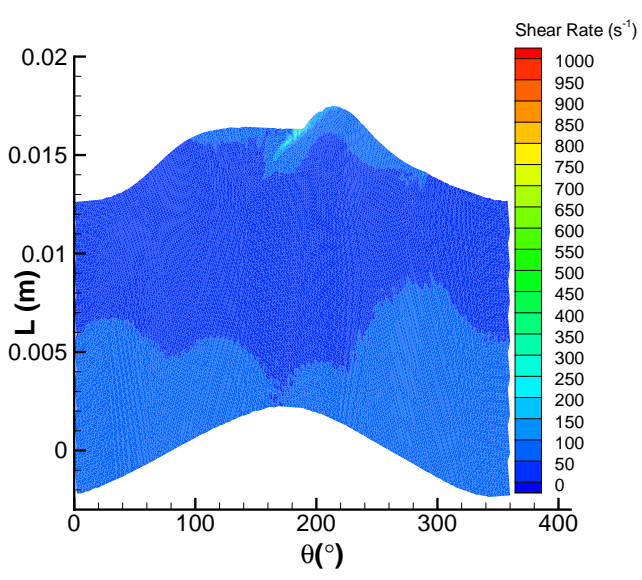

(b)

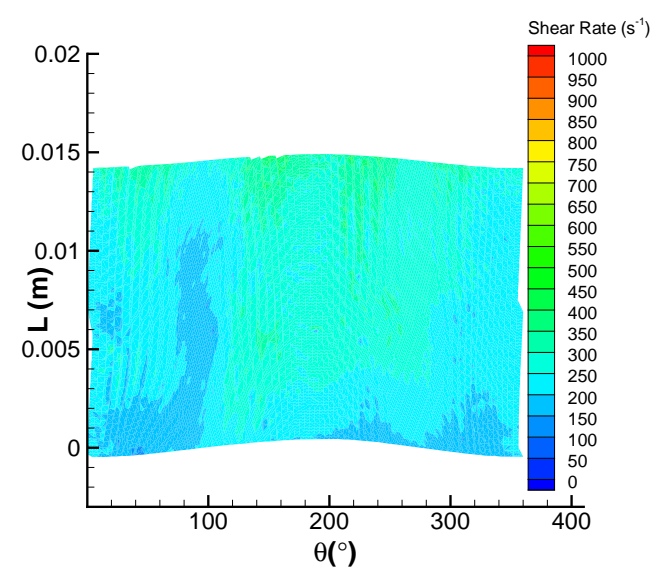

(d)

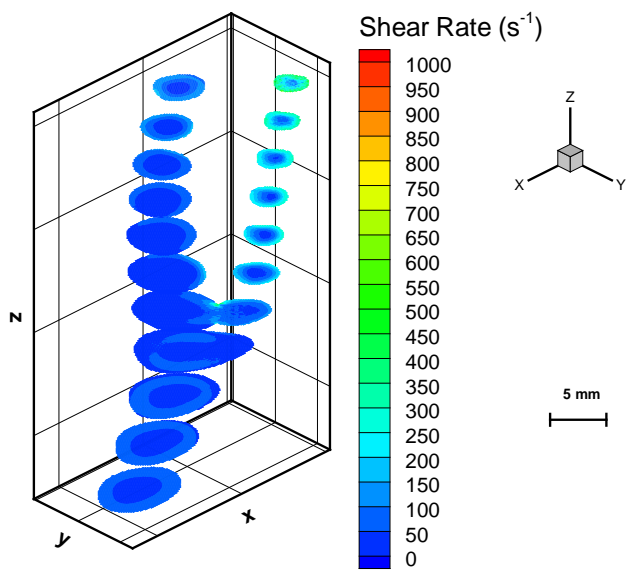

Figure 8. Near wall shear in (a) region 1, (b) region 2 and (c) region 3 at time $t=0.03$ and shear rate in the (d) carotid bifurcation

Figures 8 - 13 show the results for $\dot{\gamma}$ in the carotid artery bifurcation at the times indicated in figure 2. In these figures, the (a) - (c) figures show the wall $\dot{\gamma}$ in regions 1 , 2 and 3 respectively. The (d) figures shows the slice decomposition of $\dot{\gamma}$. Animations $4-7,{ }^{*}$ LINK $^{* *}$ show the shear variations in these regions over the whole pulse cycle.

It is observed that in general the shear rate in the ECA is much higher than that observed in the CCA and ICA. This is particularly true at the time of peak velocity, figure 9 (b) where the maximum shear is $7000 \mathrm{~s}^{-1}$. This is much greater than the 
(a)

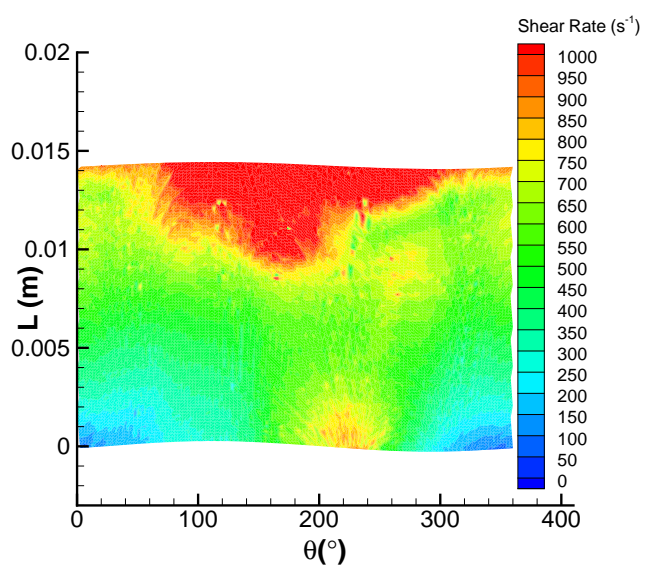

(b)

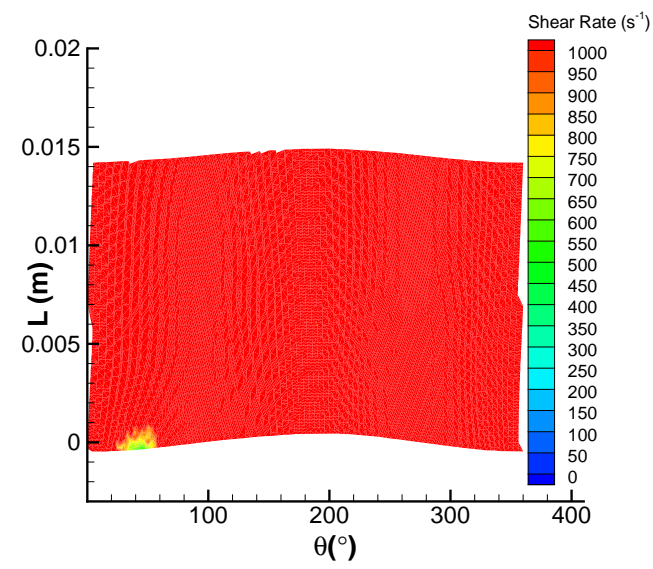

(d)

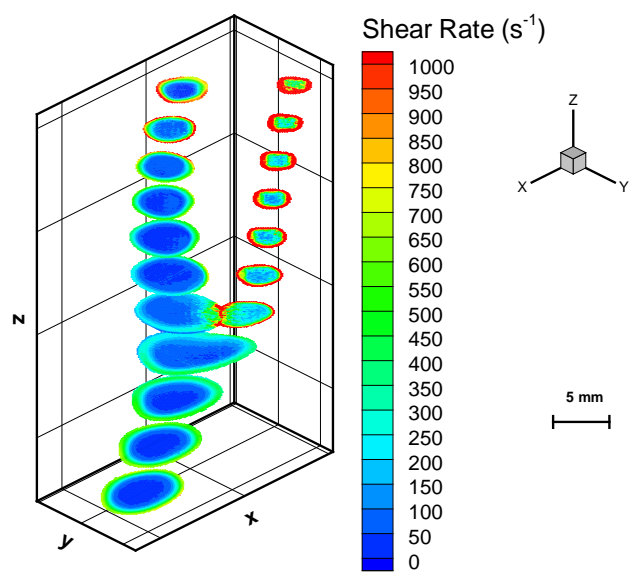

(c)

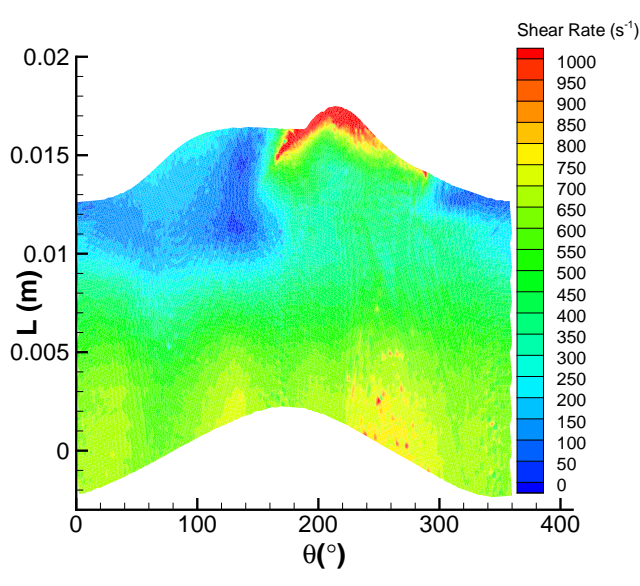

Figure 9. Near wall shear in (a) region 1, (b) region 2 and (c) region 3 at time $t=0.10$ and shear rate in the $(\mathrm{d})$ carotid bifurcation

observed shear for the rest of the artery for the whole pulse, which in general remains below $\dot{\gamma}=1000 \mathrm{~s}^{-1}$. The high shear rate seen at $t=0.1 \mathrm{~s}$ occurs along the whole wall of the ECA. It can be seen that this higher shear rate extends into the central region of the artery, figure $9(\mathrm{~d})$.

The highest shear rates in the body of the blood flow generally occur near the walls of the artery. However, a region of low wall shear rate is seen on the outer wall of the ICA, $\left(\theta \in\left(0^{\circ}, 100^{\circ}\right) \cup\left(280^{\circ}, 360^{\circ}\right), L \in(0.005,0.008)\right)$, and in the adjoining region of the bifurcation. This low region is apparent even at the time of peak velocity, figures 9 (a) and (c). A similar region is observed at the base of the ECA, although this region is not apparent at the time of peak velocity.

Higher shear rates in the central regions of the artery are observed at times of $t=0.17 \mathrm{~s}-t=0.31 \mathrm{~s}$, figures $10(\mathrm{~d})-12(\mathrm{~d})$ respectively. These higher shear rates are associated with the movement of the velocity peak observed in figure 5 of [9]. High shear rates associated with the near wall velocity seen in figure 5 of [9] are also observed on the inner wall in this region of the ICA. Low near wall shear rate is observed on the 
(a)

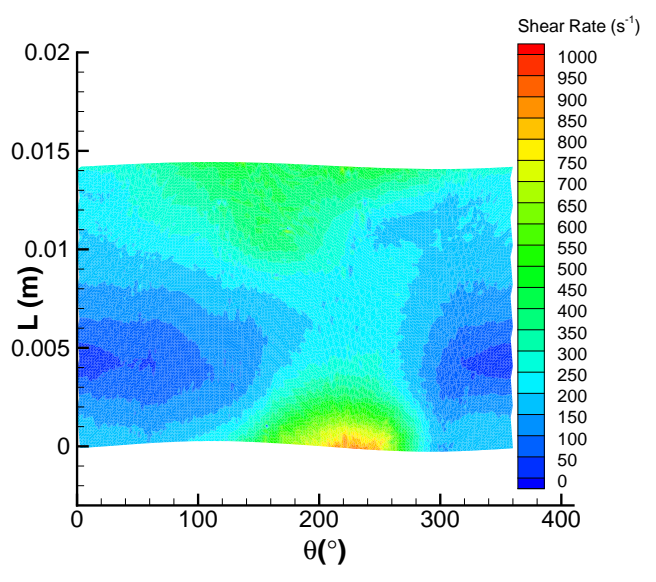

(b)

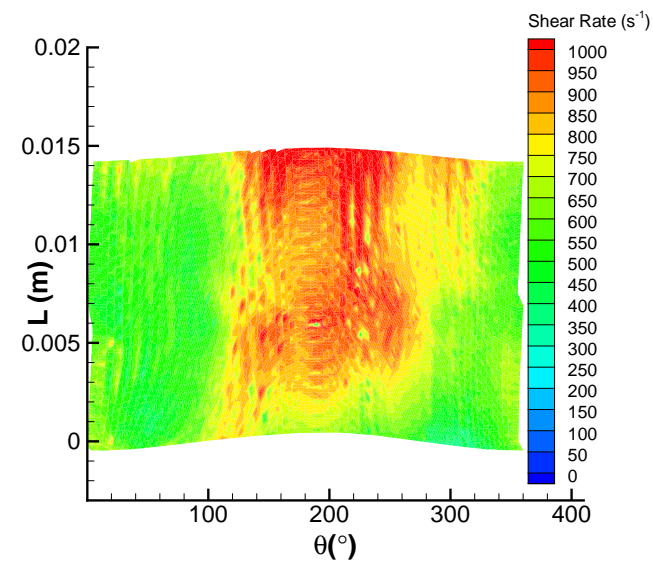

(d)

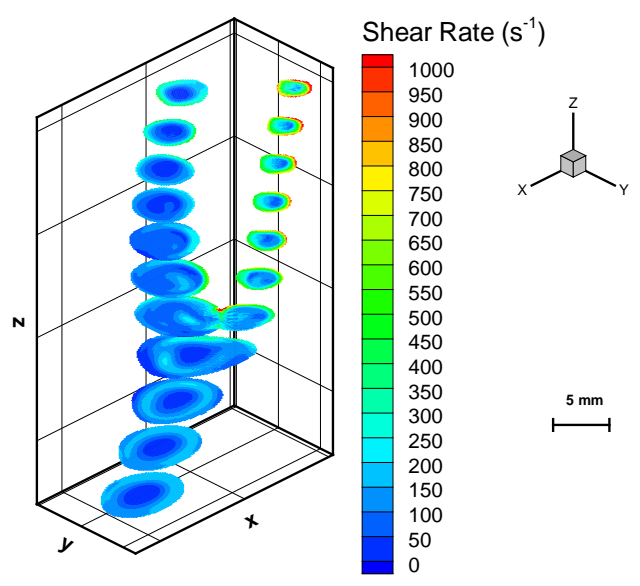

(c)

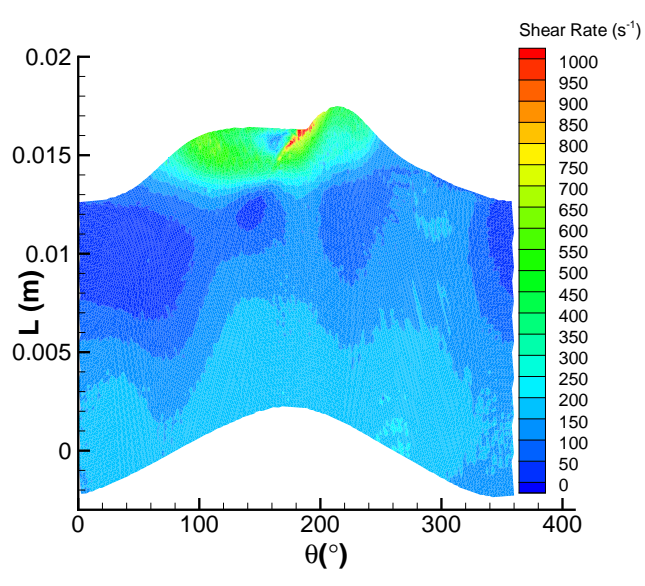

Figure 10. Near wall shear in (a) region 1, (b) region 2 and (c) region 3 at time $t=0.17$ and shear rate in the (d) carotid bifurcation

outer wall.

This low region of wall shear rate in the ICA is confined to the lower regions near the bifurcation for most of the period, although low wall shear rate over most of the ICA is observed at $t=0.24 \mathrm{~s}$, figure 11 . The peak shear rate in the ICA was $2100 \mathrm{~s}^{-1}$, at time $t=0.1 \mathrm{~s}$, less than a third of that found in the ECA at the corresponding time. This is due to the relatively lower velocities in the ICA as well as the ICA's larger diameter.

The CCA has moderate near wall shear rates for most of the pulse cycle. At times of low velocity, figures 11 - 13, the near wall shear rate is relatively low compared to that seen in the ECA. It can be seen in figure 12 that the reduction in the velocity as the blood flow enters the bifurcation region also results in low wall shear rate on the upper edges of the CCA.

A similar effect can be seen at time $0.17 \mathrm{~s}$, figure 10 , where the splitting of the flow into the ICA and ECA as it streams out of the CCA results in the low wall shear rate on the lower wall of the ICA and also precipitates the movement of the velocity peak 
(a)

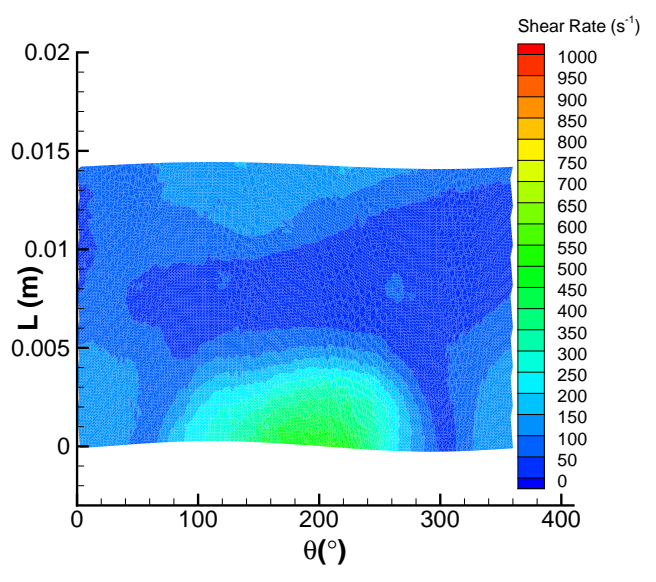

(b)

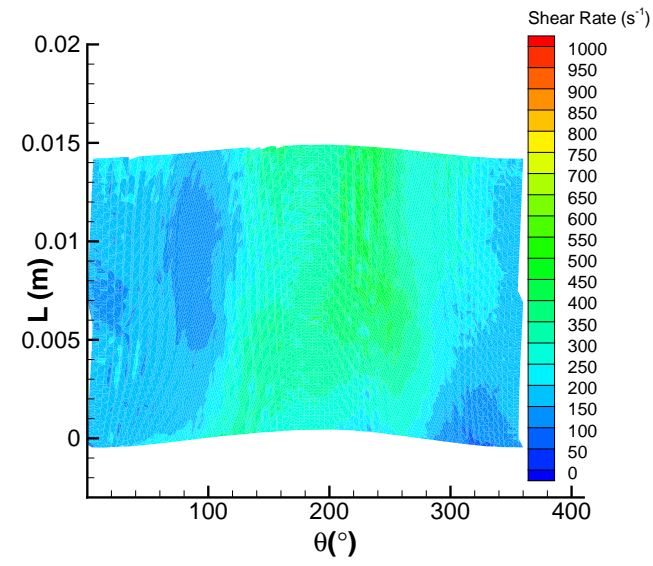

(d)

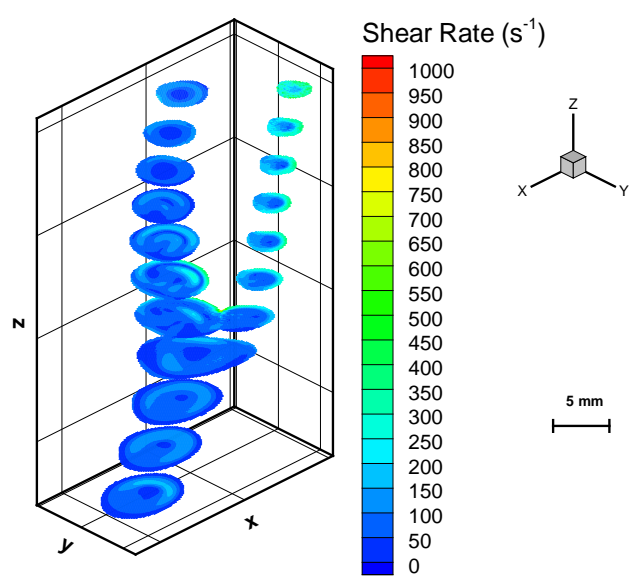

(c)

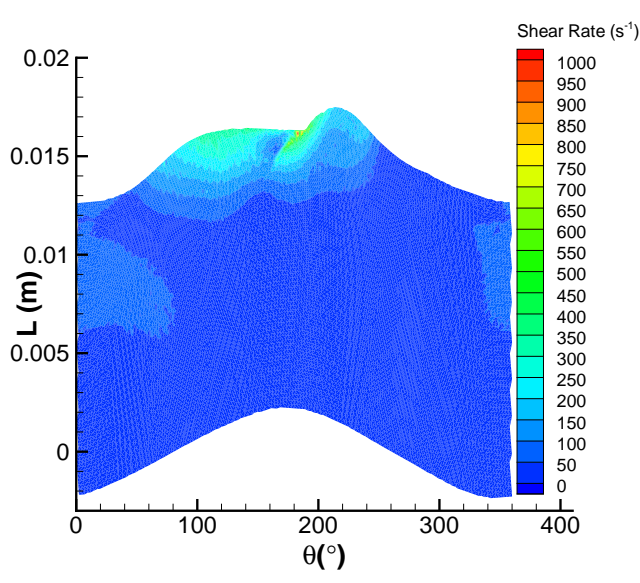

Figure 11. Near wall shear in (a) region 1, (b) region 2 and (c) region 3 at time $t=0.24$ and shear rate in the (d) carotid bifurcation

observed later in the period [9].

Relatively higher wall shear rate can be seen in region 3 where the ICA and ECA bifurcate from each other. This is observed in the middle top region of figures 8 - 13 (c), $\left(\theta \in\left(160^{\circ}, 260^{\circ}\right), L \in(0.014,0.018)\right)$. This arises from the way the fluid is forced into either the ICA or ECA branch of the bifurcation. This region is subjected to high wall shear rate and high velocity for most of the pulse cycle.

The shear rate in each region is roughly symmetric about $\theta=180^{\circ}$. In regions 1 and 2 this approximate symmetry is clear and the shear rate changes with both $\theta$ and $L$. In region 3 , below the bifurcation, the shear rate depends predominantly on $L$ over the majority of the period with a dependence on $\theta$ only apparent at certain phases. It is evident that the shear rate is also dependent on features other than the local $L$ and $\theta$ coordinates; for example the high shear rate at the bifurcation observed in region 3 (figures $8-13(\mathrm{c})$ ) for $L \geq 0.015 \mathrm{~m}$. 
(a)

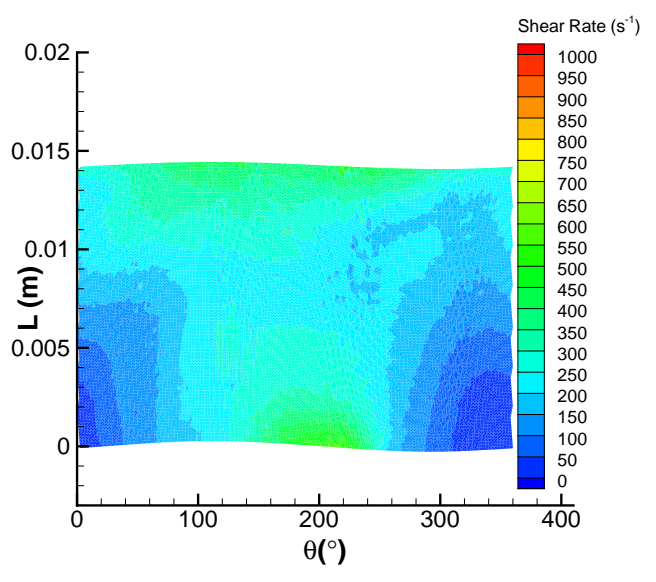

(b)

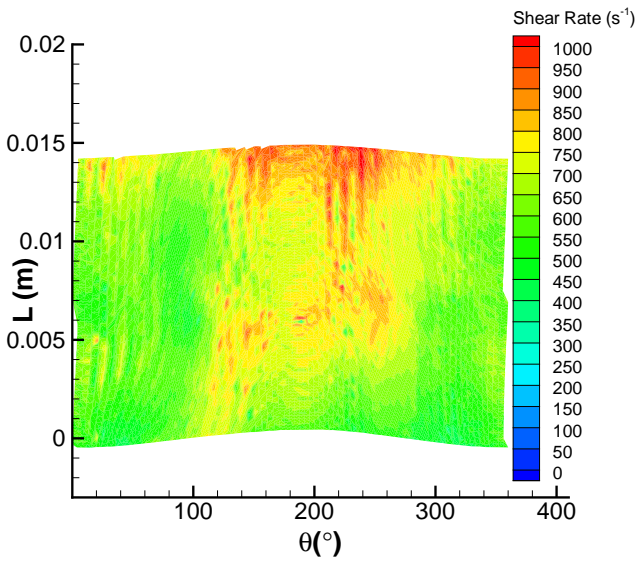

(d)

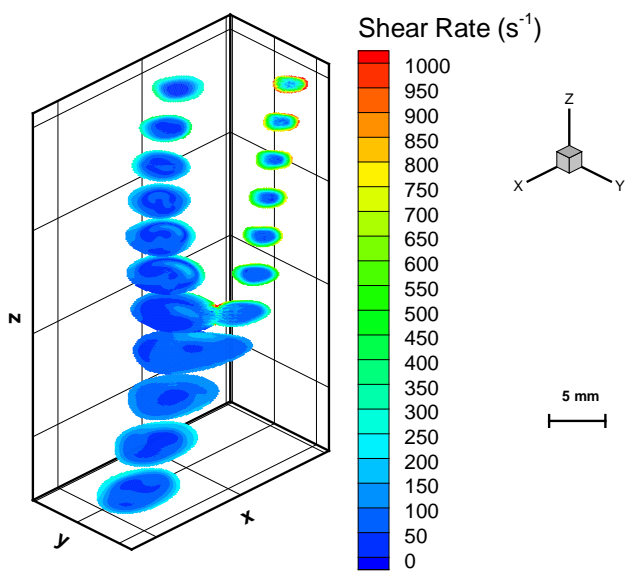

(c)

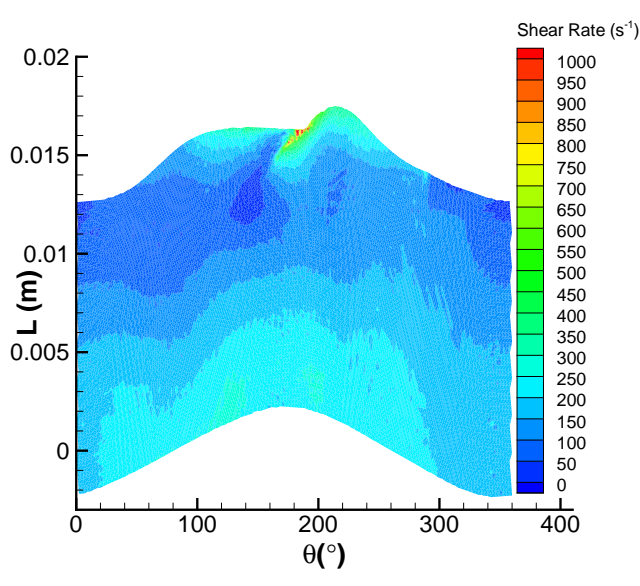

Figure 12. Near wall shear in (a) region 1, (b) region 2 and (c) region 3 at time $t=0.31$ and shear rate in the (d) carotid bifurcation

\section{Discussion}

The behaviour of the shear rate in the $3 \mathrm{D}$ model agrees well with the characteristics observed in physiological models [3, 5, 22] as well as those seen in previous 2D simulations $[13,14]$. Haemodynamic features associated with atherosclerotic cardiovascular disease have been identified. Low wall shear rate is seen in the bifurcation region near the base of the ICA for most of the pulse period. The same area is also known to experience low blood velocity and circulating flow [9]. This region is known to be prone to atherosclerosis and the haemodynamic conditions observed here are consistent with this. High shear near the wall of the bifurcation is also observed, extending relatively higher wall shears along the walls of the ECA for the duration of the pulse period, indicating that this is a region that would not be prone to atherosclerosis.

The approximate symmetry about $\theta=180^{\circ}$ in the shear rate (figures 8 - 13 (a) (c) and animations $4-6{ }^{* *}$ LINK $^{* *}$ ) mirrors that observed in the radius in region 3 , below the bifurcation, in figure 6 (c). This suggests that the distribution of the wall 
(a)

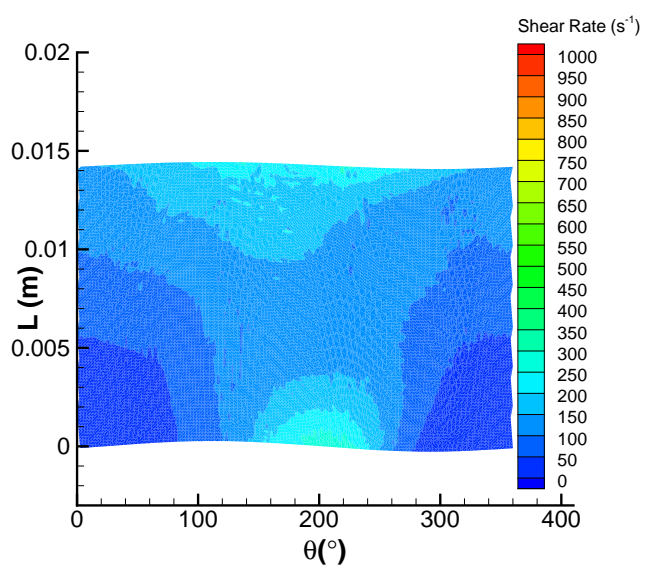

(b)

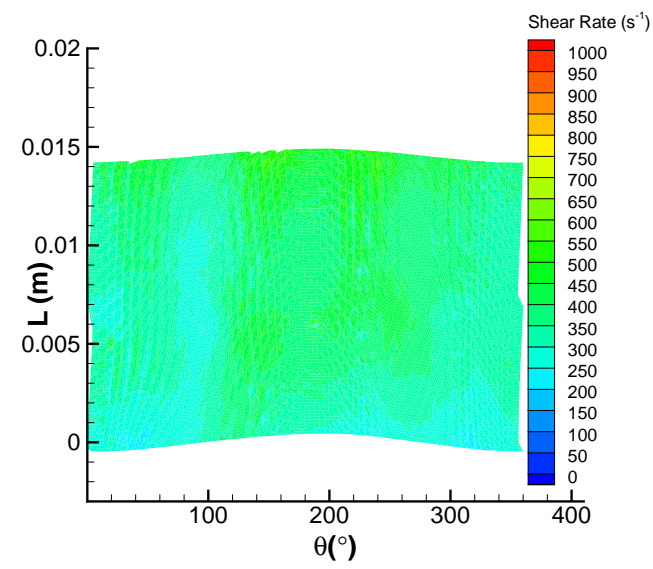

(d)

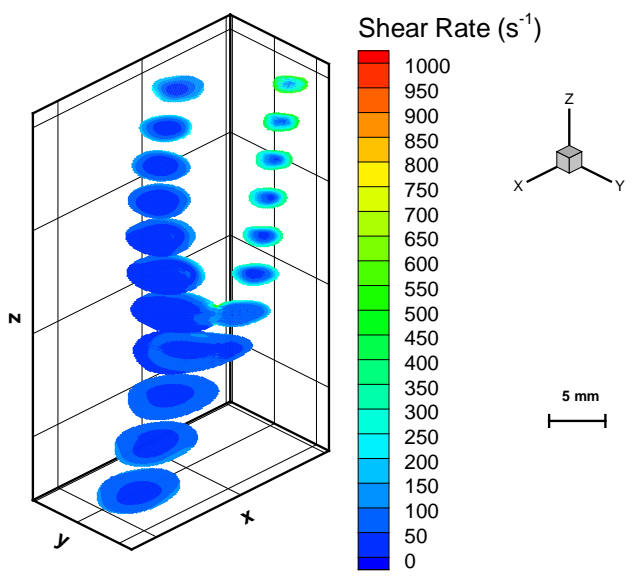

(c)

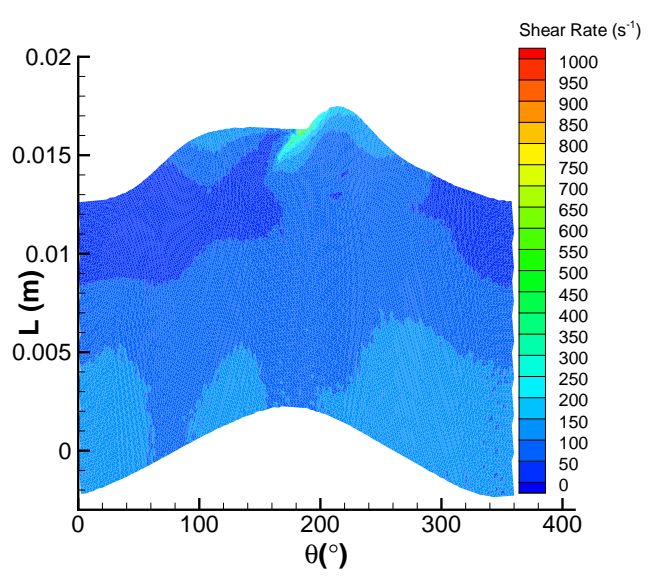

Figure 13. Near wall shear in (a) region 1, (b) region 2 and (c) region 3 at time $t=0.60$ and shear rate in the (d) carotid bifurcation

shear, and therefore the susceptibility of the artery to atherosclerosis, just above the bifurcation is influenced more by the geometry of the artery below the bifurcation, than the local geometry.

Details of the velocity field in the region of the carotid bifurcation [9] combined with the local shears presented here, provide a comprehensive description of the blood flow. Thus the haemodynamic conditions can be identified in regions prone to atherosclerosis as well as areas where stenosis growth is uncommon. It is hoped that this will lead to an improved understanding of the complex relationship between blood flow and the progression of atherosclerosis. Further work is required to study how the haemodynamics change due to the presence and growth of a stenosis.

The LBM has been shown to lend itself easily to analyzing the near wall stress and modelling the complicated flow patterns encountered in the human carotid artery. This makes it ideal for the study of the haemodynamic influences on the progression of atherosclerosis. As with all numerical approaches, assumptions in the model have to be considered when interpreting the results. A number of these are discussed below. 
The simulations were performed using an artery model with rigid boundaries; however, the carotid artery is known to change diameter during the cardiac cycle. Further work is required to determine the extent to which this influences the results. A system for incorporating elastic and moving boundaries has been proposed [23, 24] and applied to steady and pulsatile flows in elastic vessels with similar pressure-radius relationships to pulmonary vessels.

Another feature is the method used to implement the velocity waveform. This was implemented at the base of the artery with the velocity uniform across the cross section except for a small boundary layer region. This velocity profile is more typical of a vessel with a larger Womersley parameter, such as the Aorta. We note that this velocity profile is implemented at the inlet some distance from the region of interest shown in figure 1(a). The velocity results presented in [9] show a more parabolic like profile at the base of the region of interest. The volume between the base of the artery and the region of interest acts as a buffer region in which the velocity profile converts to the 'correct' one for the artery geometry. It is felt that the approach of simulating a larger region of the artery with a buffer region at the base provides a greater level of accuracy compared to implementing some expected velocity profile at the base of the region of interest.

It was also assumed that blood can be treated as a Newtonian fluid. It is known that blood is actually non-Newtonian with an apparent viscosity which is a function of the local shear. The non-Newtonian nature of blood can be incorporated in the LBM [25] and may be important for smaller arteries. For the carotid artery previous work suggests that errors in applying a Newtonian model are generally small and not significant compared to differences between alternative non-Newtonian models [21].

\section{Conclusion}

The 3D carotid artery model has been used to study the properties of the shear and the near wall shear, represented by the shear rate $\dot{\gamma}$, over a pulse cycle of Newtonian blood flow.

It has been shown that the shear rate $\dot{\gamma}$ and the von Mises effective shear $\sigma_{\text {eff }}$ exhibit similar near wall features. High shears are observed to be localised near the artery wall for most of the pulse cycle. The highest shears are observed in the ECA. Some higher shears are observed in the central regions of the bifurcation and the lower regions of the ICA due to movement of the spatial position of the velocity peak induced by the bifurcating artery.

The method of near wall shear analysis allowed for regions of low shear to be identified on the walls of the arteries throughout the pulse cycle. The identified low shear regions correspond well with regions known to be susceptible to atherosclerosis, namely the outer bifurcation walls and the lower ICA. The ability to map the wall shear distribution in this manner could have further applications in studying and identifying atherosclerotic prone arteries, the study of the forces acting on plaques that cause them to rupture and the study of the shear related behaviour of endothelial cells. Further, 
combined with the velocity results presented in [9], a full picture of the haemodynamic properties of the blood in the region of interest was obtained.

\section{Acknowledgments}

The authors would like to acknowledge the technical assistance of N. Gaywood, A. Saint and S. Ryan. This work was also partially supported by Sigma Xi Grant no. 10040015 and the Australian Postgraduate Award (APA), this assistance is gratefully acknowledged.

\section{References}

[1] Murray C J L and Lopez A D. The global burden of disease: a comprehensive assessment of mortality and disability from diseases, injuries, and risk factors in 1990 and projected to 2020. Harvard school of public health MA, 1996.

[2] Caro C G. Vascular fluid dynamics and vascular biology and disease. Math. Meth. Appl. Sci., 24:1311-1324, 2001.

[3] Malek A M, Alper S L, and Izumo S. Hemodynamic Shear Stress and Its Role in Atherosclerosis. J. Amer. Med. Assoc., 282:2035-2042, 1999.

[4] Asakura T and Karino T. Flow Patterns and Spatial Distribution of Atherosclerotic Lesions in Human Coronary Arteries. Circ. Res., 66:1045-1066, 1990.

[5] Gnasso A, Irace C, Carallo C, De Franceschi M S, Motti C, Mattioli P L, and Pujia A. In Vivo Association Between Low Wall Shear Stress and Plaque in Subjects With Asymmetrical Carotid Atherosclerosis. Stroke, 28:993-998, 1997.

[6] Chen S and Doolen G D. Lattice boltzmann method for fluid flows. Ann. Rev. Fluid Mech., 30:329-364, 1998.

[7] Succi S. The Lattice Boltzmann Equation for Fluid Dynamics and Beyond. Oxford University Press, 2001.

[8] Wolf-Gladrow D A. Lattice-Gas Cellular Automata and Lattice Boltzmann Models, An introduction. Springer, 2000.

[9] J Boyd and J M Buick. Three-dimensional modelling of the human carotid artery using the lattice Boltzmann method. I: Model and velocity analysis. submitted.

[10] Boyd J, Ryan S J, and Buick J M. A parallel Lattice Boltzmann Model of a Carotid Artery. American Institute of Physics Conference Proceedings, 2008. In press.

[11] Holdsworth D W, Norley C J D, Frayne R, Steinman D A, and Rutt B K. Characterization of common carotid artery blood-flow waveforms in normal human subjects. Physiol. Meas., 20:219-240, 1999.

[12] Guo Z, Zheng C, and Shi B. An extrapolation method for boundary conditions in lattice Boltzmann method. Phys. Fluids, 14(6):2007-2010, 2002.

[13] Boyd J, Buick J M, Cosgrove J A, and Stansell P. Application of the lattice Boltzmann model to simulated stenosis growth in a two-dimensional carotid artery. Phys. Med. Biol., 50:4783-4796, 2005 .

[14] Boyd J and Buick J M. Comparison of Newtonian and non-Newtonian flows in a two-dimensional carotid artery model using the lattice Boltzmann method. Phys. Med. Biol., 52(20):6215-6228, 2007.

[15] Boyd J, Buick J M, Cosgrove J A, and Stansell P. Application of the lattice Boltzmann method to arterial flow simulation: Investigation of boundary conditions for complex arterial geometries. Australas. Phys. Eng. Sci. Med., 27(4):207-212, 2004.

[16] Artoli A M. Mesoscopic Computational Haemodynamics. Ponsen \& Looijen, Wageningen, 2003. 
[17] Geiringer H. Recent results in the theory of plasticity. In von Karmen R, editor, Advances in applied Mechanics III. Academic Press, 1953.

[18] Amtec. Tecplot User's Manual, Version 10. Amtec Engineering, Inc., Bellevue Washington, 2003.

[19] Ku D N, Giddens D P, Zarins C K, and Glagov S. Pulsatile Flow and Atherosclerosis in the Human Carotid Bifurcation: Positive Correlation between Plaque Location and Low and Oscillating Shear Stress. Arteriosclerosis, 5(3):293-302, 1985.

[20] Perktold K, Resch M, and Florian H. Pulsatile Non-Newtonian Flow Characteristics in a ThreeDimensional Human Carotid Bifurcation Model. J. Biomech. Eng., 113:464-475, 1991.

[21] Boyd J, Buick J M, and Green S. Analysis of the Casson and Carreau-Yasuda Non-Newtonian Blood Models in Steady and Oscillatory Flows Using the Lattice Boltzmann Method. Phys. Fluids, 19(9):093103, 2007.

[22] Quarteroni A, Tuveri M, and Veneziani A. Computational vascular fluid dynamics: problems, models and methods. Comput. Visual. Sci., 2:163-197, 2000.

[23] Fang H, Wang Z, Lin Z, and Liu M. Lattice Boltzmann simulation of viscous fluid systems with elastic boundaries. Phys. Rev. E, 57(1):R25-R28, 1998.

[24] Fang H, Wang Z, Lin Z, and Liu M. Lattice Boltzmann method for simulating the viscous flow in large distensible blood vessels. Phys. Rev. E, 65:051925:1-11, 2002.

[25] Boyd J, Buick J M, and Green S. A second order accurate lattice Boltzmann non-Newtonian flow model. J. Phys. A-Math. Gen., 39:14241-14247, 2006. 